Journal de la Société des américanistes

Journal de la Société

des américanistes

$96-2 \mid 2010$

tome $96, \mathrm{n}^{\circ} 2$

\title{
Guy Stresser-Péan (1913-2009)
}

\section{Dominique Michelet}

\section{OpenEdition}

Journals

Édition électronique

URL : https://journals.openedition.org/jsa/11591

DOI : 10.4000/jsa. 11591

ISSN : $1957-7842$

Éditeur

Société des américanistes

Édition imprimée

Date de publication : 5 décembre 2010

Pagination : 289-292

ISSN : 0037-9174

\section{Référence électronique}

Dominique Michelet, «Guy Stresser-Péan (1913-2009)», Journal de la Société des américanistes [En ligne], 96-2 | 2010, mis en ligne le 22 juillet 2010, consulté le 03 septembre 2022. URL : http:// journals.openedition.org/jsa/11591 ; DOI : https://doi.org/10.4000/jsa.11591 


\section{NÉCROLOGIES}

\section{Guy STRESSER-PÉAN (1913-2009)}

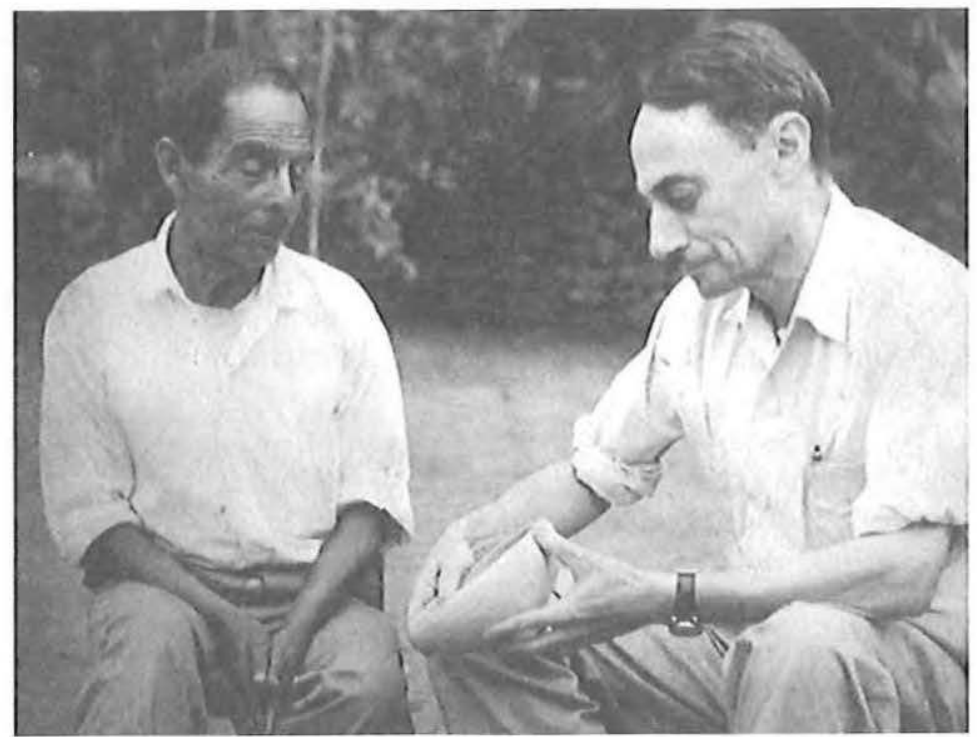

Guy Stresser-Péan, en 1952, avec Martiniano Castillo du village de Chililico ( $\mathrm{M}^{\circ}$ Huejutla, Hidalgo, Mexique).

Guy Stresser-Péan s'est éteint le 8 novembre 2009 à son domicile de Mexico, ville où il était arrivé, jeune diplômé de l'Institut d'ethnologie de Paris, mandaté par Paul Rivet et pourvu d'une bourse, soixante-treize ans plus tôt presque jour pour jour (très exactement le 5 décembre $1936^{1}$ ).

À moins de deux semaines de distance, sa disparition a suivi celle de son aîné - ils avaient en fait moins de trois ans de différence - et ami, Claude Lévi-Strauss, dont la place dans le monde scientifique avait été très différente de la sienne, mais qui avait, pour l'œuvre de Guy Stresser-Péan, une réelle admiration. On n'en citera pour preuve, parmi plusieurs témoignages, que ces quelques mots extraits d'une lettre manuscrite qu'il lui adressa le 20 septembre 2005 et relatifs au livre que Guy Stresser-Péan lui avait fait parvenir peu auparavant, Le Soleil-Dieu et le Christ. La christianisation des Indiens du Mexique vue de la Sierra de Puebla (l'Harmattan, Paris, 2005 ${ }^{2}$ ) : « C'est un trésor d'observations et de réflexions originales que je vous suis reconnaissant de m'avoir envoyé. J'admire que vous ayez mené à bien cette grande entreprise malgré les fatigues de l'âge. Plus vieux que vous, certes, j'ai renoncé aux livres " ${ }^{3}$. 
De fait, c'est un des points de la trajectoire professionnelle de Guy StresserPéan dont on ne finira jamais de s'étonner : sa longévité et son extraordinaire richesse jusque - et même surtout, peut-on dire - dans ses dernières années. Pendant des décennies, en effet, celui qui était pourtant internationalement reconnu comme l'un des meilleurs "huastécologues ${ }^{4}$ », dont la compétence allait des temps précolombiens jusqu'à l'époque contemporaine ${ }^{5}$, n'avait publié que quelques articles, souvent importants, mais demeurés parfois peu connus ${ }^{6}$. Le premier des sept livres qu'on doit à Guy Stresser-Péan et qui sont tous essentiels ${ }^{7}$, chacun dans son domaine, San Antonio Nogalar: La Sierra de Tamaulipas et la frontière nord-est de la Mésoamérique (Mission archéologique et ethnologique française au Mexique, Mexico, $1977^{8}$ ) parut quand son auteur avait soixante-trois ans ; le deuxième, El arado criollo en México y América central (CEMCA/IFAL/ORSTOM, Mexico, 1988) alors qu'il avait soixante-quatorze ans et les cinq suivants entre son quatre-vingt-unième et son quatre-vingtdouzième anniversaire.

Il n'est pas douteux que, lorsque Guy Stresser-Péan accepta de créer; en 1961, et de diriger, pendant plus de seize ans, ce qui s'appela d'abord la Mission archéologique et ethnologique française au Mexique (MAEF), devenu, au début des années 1980, CEMCA (Centre français d'études mexicaines et centraméricaines), ce rôle le détourna un peu de la recherche et beaucoup de l'écriture... sinon celle de multiples rapports. Mais l'œuvre qui fut la sienne à cette période à la tête de la MAEFM eut, quoi qu'elle lui ait coûté sur un plan personnel, une importance radicale et une postérité qui n'est pas près de s'éteindre. Le CEMCA, institut français de recherche à l'étranger du ministère des Affaires étrangères et européennes ${ }^{9}$, demeure un centre actif de recherche et le lieu privilégié de riches coopérations scientifiques franco-mexicaines et franco-centraméricaines portant sur des aspects multiples des réalités des pays partenaires. Et c'est aussi très largement à Guy Stresser-Péan que l'on doit l'existence aujourd'hui d'une vraie, large et dynamique communauté de chercheurs professionnels français travaillant principalement dans toutes les disciplines que recouvre aux Étas-Unis le terme " anthropologie».

Cela dit, à la production si longtemps différée d'ouvrages scientifiques que tous attendaient de Guy Stresser-Péan, c'est Miguel León-Portilla qui donne la bonne et seule véritable explication. Dans le prologue qu'il rédigea pour le volume Viaje a la Huasteca, celui-ci écrit en effet avec pertinence : "Sólo cuando [él] considera[ba] haber agotado los recursos a su alcance para ahondar en el asunto de que se ocupa[ba], decid[ia] dar a conocer los resultados de su trabajo". Guy Stresser-Péan était effectivement un authentique érudit, ce qui avait pour corollaire qu'il s'imposait toujours de chercher à rassembler, sur tous les sujets qu'il abordait, toute la documentation possible, livresque et de terrain à la fois. Par ailleurs, c'était un homme dont la formation avait été large, multidisciplinaire dirait-on aujourd'hui puisqu'il s'était spécialisé, d'un côté, en sciences sociales, 
comme étudiant de Mauss en particulier, mais aussi en sciences de la nature. Et cela, naturellement, le prédisposait à envisager tous les aspects de ses sujets d'étude et à les traiter avec un même souci d'exhaustivité. Les livres qu'il publia, à partir donc d'un âge avancé, illustrent bien l'étendue de ses centres d'intérêts ${ }^{10}$. Trois concernent des recherches archéologiques qui eurent pour cadre les sites de San Antonio Nogalar, sur la frontière nord-est de la Mésoamérique, et de Tamtok, établissement capital au cœur du pays huastèque. Deux autres sont des ouvrages d'ethnohistoire consacrés, pour l'un, au codex de Xicotepec, qu'il découvrit et analysa magistralement, et, pour l'autre, aux lienzos d'Acaxochitlan, dont il a dévidé tout le sens. Les deux derniers enfin, d'ordre ethnographique, font chacun le tour d'une question, d'envergure bien différente, d'ailleurs : l'araire en Mésoamérique (son introduction date de l'époque coloniale); les processus d'évangélisation des Indiens de la Sierra Norte de Puebla et la religion de ces derniers jusqu'au milieu du $\mathrm{xx}^{\mathrm{e}}$ siècle. Le Soleil-Dieu et le Christ, qui est consacré à ce dernier sujet, particulièrement complexe, est certainement l'ouvrage le plus représentatif de l'homme et du chercheur que fut Guy Stresser-Péan ${ }^{11}$. On l'y découvre partagé entre l'immersion dans les sociétés indiennes [sub]contemporaines et le travail de remontée dans le temps qui lui permet de comprendre pourquoi et comment les Indiens d'aujourd'hui ou d'hier sont ce qu'ils sont [ou ce qu'ils étaient]. Partagé aussi entre une présentation et une analyse générales des faits (des rituels principalement, en l'occurrence) et une attention méticuleuse, voire pointilliste, à tel ou tel détail précis (les deux grandes catégories de tambours hérités du monde d'avant la Conquête, par exemple), mais jamais déplacée, parce que tout a du sens et mérite qu'on s'en préoccupe. On le découvre enfin s'eflaçant toujours derrière ces hommes et ces femmes indiens, cette partie de l'humanité à la connaissance de laquelle il œuvrait. Car, si Guy Stresser-Péan a su faire parler les tessons et les cailloux, c'est bien d'abord aux hommes, et aux Indiens tout spécialement, qu'en réalité il a toujours préféré s'adresser. Pour tenter de comprendre leurs modes de vie et leurs façons de penser le monde, assurément, mais aussi, sans aucun doute, tout simplement par empathie profonde. Qui a eu le privilège de l'accompagner sur le terrain ethnographique, même fugitivement comme ce fut mon cas, peut en témoigner: l'érudit qu'il était aux yeux de beaucoup était, plus fondamentalement encore, dans les villages qu'il fréquentait, un homme ouvert aux autres et passionné par leur altérité. Quand il retrouvait tel ou tel de ses « informateurs », les plus proches en tout cas - ensemble ils donnaient bien plus l'impression d'être des amis complices -, le monde aurait pu s'écrouler : rien ne comptait plus que la reprise d'un dialogue, mais qui était aussi une enquête, et dont on avait d'ailleurs l'impression qu'il n'avait jamais été interrompu... 


\section{NOTES}

1. Pour une biographie de Guy Stresser-Péan, du moins des soixante-quinze premières années de sa vie, on peut se référer à l'article que l'auteur de la présente notice lui avait consacré : « Un savant, Guy Stresser-Péan ", in Dominique Michelet (éd.), Enquêtes sur l'Amérique moyenne. Mélanges offerts à Guy Stresser-Péan, INAH/CONACULTA/CEMCA, Mexico, 1989, pp. 15-25. Un autre texte, nettement plus récent, éclaire bien, quant à lui, la carrière autant que la personnalité de Guy Stresser-Péan : il s'agit de la transcription d'entretiens qu'il eut avec Guilhem Olivier en 2003 et 2004 : « Pláticas con Guy Stresser-Péan (entrevistas realizadas por Guilhem Olivier) ", in Guilhem Olivier (éd.), Viaje a la Huasteca con Guy Stresser-Péan, Fondo de Cultura Económica/CEMCA, Mexico, 2008, pp. 27-65.

2. Signalons ici qu'à la médiocre édition française de cette somme - parue chez l'Harmattan, faute d'avoir retenu en France l'attention d'une maison d'édition digne de ce nom, conformément à l'adage évangélique selon lequel "nul n'est prophète en son pays »-a succédé heureusement une version anglaise beaucoup mieux éditée et nettement plus élégante : The Sum-God and the savior. The christianization of the Nahua and Totonac in the Sierra Norte de Puebla, Mexico, University Press of Colorado, Boulder, 2009.

3. Je remercie vivement Claude Stresser-Péan de m'avoir communiqué une copie de ce courrier ainsi que celle d'un autre, un peu antérieur.

4. Ou spécialistes de la Huasteca, région du Mexique située au nord de la côte du Golfe.

5. C'est lui qui avait été chargé de rédiger un des articles de référence pour le Handbook of Middle American Indians, "Ancient Sources on the Huasteca », lequel fut intégré dans le tome 11 (pp. 582 602) de l'ouvrage - publié en 1971.

6. Fort heureusement, la plupart d'entre eux ont été réédités récemment et en version espagnole quand ils ne l'étaient pas initialement, dans le bel ouvrage coédité par le Fondo de Cultura Económica et le CEMCA qu'a coordonné Guilhem Olivier (2008) : voir ci-dessus note 2.

7. La Huasteca est l'objet, depuis les années 1990, d'études dont le développement a été spectaculaire, et force est de constater que pratiquement tous les chercheurs qui s'intéressent désormais à ce champ reconnaissent la dette qu'ils ont vis-à-vis des travaux de Guy Stresser-Péan.

8. Réédité en espagnol en 2000 grâce à une coédition entre le CIESAS, le Colegio de San Luis et l'Universidad Autónoma de Tamaulipas ( San Antonio Nogalar. La Sierra de Tamaulipas y la frontera de la Mesoamérica).

9. Et également, depuis le $1^{\text {er }}$ janvier 2010 , unité de service et de recherche du CNRS.

10. Il ne faudrait cependant pas oublier l'importante auvre filmographique qui fut celle de Guy Stresser-Péan : la plupart de ses films (une demi-douzaine), qui sont autant de témoignages ethnographiques de première importance et de grande qualité, ont été réalisés avec son épouse, Claude.

11. Alfredo López Austin, qui est l'auteur du prologue de ce volume, met particulièrement bien en exergue ce qu'il est et tout ce qu'il apporte. 